\title{
Large database utilization in health outcomes research in pancreatic cancer: an update
}

\author{
Anna Torgeson ${ }^{1}$, Randa Tao $^{1}$, Ignacio Garrido-Laguna ${ }^{2}$, Benjamin Willen ${ }^{3}$, Amy Dursteler ${ }^{4}$, Shane Lloyd $^{1}$ \\ ${ }^{1}$ Department of Radiation Oncology, ${ }^{2}$ Department of Internal Medicine, University of Utah, Salt Lake City, Utah, USA; ${ }^{3}$ Wright State University, \\ Boonshoft School of Medicine, Dayton, Ohio, USA; ${ }^{4}$ The University of Texas Medical School, Houston, Texas, USA \\ Contributions: (I) Conception and design: S Lloyd, A Torgeson; (II) Administrative report: S Lloyd; (III) Provision of study materials: B Willen, \\ A Dursteler, A Torgeson, S Lloyd, I Garrido-Laguna; (IV) Collection and assembly of data: All authors; (V) Data analysis and interpretation: All \\ authors; (VI) Manuscript writing: All authors; (VII) Final approval of manuscript: All authors. \\ Correspondence to: Shane Lloyd, MD. 1950 Circle of Hope, Rm 1570, Salt Lake City, Utah 84112, USA. Email: shane.lloyd@hci.utah.edu.
}

\begin{abstract}
We sought to review published aggregate dataset studies on pancreatic cancer in the national and international settings, discuss the advantages and disadvantages these datasets possess, and possible future directions. A combination of Google Scholar, PubMed, and MEDLINE were used with search terms "pancreatic cancer" + "resectable" + "national cancer database", "pancreatic cancer" + "unresectable" + "national cancer database" and more broadly "borderline resectable pancreatic cancer", "locally advanced pancreatic cancer", "unresectable pancreatic cancer", and "resectable pancreatic cancer". Original articles and abstracts from this search were included, including data from the Surveillance, Epidemiology, and End Results (SEER) database, National Cancer Database (NCDB), and SEER-Medicare within the United States (US), as well as international database studies. Multiple database studies have been published regarding the role for radiotherapy in resected pancreatic cancer $(n=6)$, the timing of additional therapy in resectable pancreatic cancer $(n=4)$, and the role for radiotherapy and resection in locally advanced pancreatic cancer (LAPC) ( $\mathrm{n}=4)$. Studies from both SEER and NCDB found a survival benefit to post-operative radiotherapy. In resectable pancreatic cancer, neoadjuvant treatment was found to be superior to adjuvant (NCDB). Chemoradiotherapy was found to be more beneficial than chemotherapy alone in LAPC, and patients who received highly-conformal or stereotactic body radiotherapy (SBRT) had improved survival compared to either conformal radiotherapy or chemotherapy alone. These studies also found that up to $10 \%$ of patients underwent resection, with a $90 \%$ margin-negative rate, and either one-half to one-third the risk of death of non-surgical patients. Criticism of large datasets includes lack of granularity of performance status, diagnosis, treatment, and outcomes-related data compared to properly administered prospective trials, as well as crossover between treatment arms that cannot be accounted for, and concerns over quality of data represented. The US has witnessed a growing number of comparative effectiveness studies in pancreatic cancer. When taken together, certain themes emerge that are consistent with both single-institution data and clinical trials. These studies have also provided insight into questions not readily answerable by clinical trials. However, they require caution in interpretation.
\end{abstract}

Keywords: Health outcomes research; National Cancer Database (NCDB); pancreatic cancer

Submitted Jan 31, 2018. Accepted for publication May 16, 2018.

doi: 10.21037/jgo.2018.05.15

View this article at: http://dx.doi.org/10.21037/jgo.2018.05.15

(c) Journal of Gastrointestinal Oncology. All rights reserved. 


\section{Introduction}

Though survival for all stages of pancreatic cancer has improved over the last few decades, it remains dismal (1). The mortality rate for pancreatic cancer approaches its incidence rate every year (2), and it is projected to be the second leading cause of cancer death in the world by 2030 (3). Several important clinical trials have been recently reported regarding pancreatic cancer treatment, though almost as many questions have been generated from these reports as have been answered. Though randomized studies may have the highest degree of internal validity, it is not uncommon for their external validity to be questioned even before the study has been completed. Completed studies may also seem to contradict one another, making interpretation further difficult. As such, there are still several open questions in the clinical management of patients with pancreatic cancer. These include the most appropriate timing of additional therapy with respect to resection [neoadjuvant (NAT) versus adjuvant (AT)], the role for radiotherapy in resected pancreatic cancer, the role for radiotherapy in locally advanced (LAPC) and borderline resectable pancreatic cancer (BRPC), and the possibility of surgical resection after primary therapy for LAPC patients.

Given the expense and time required to answer such a large number of clinical questions with phase III randomized trials, and the limited statistical power and generalizability of single-institution data, investigators have increasingly turned to large aggregated datasets to explore these questions. These large database series are particularly useful in describing the epidemiology and trends in incidence, treatment, survival, and local control of different cancers, as well as changes in practice patterns over time. More recently, in the United States (US), these datasets have been widely used for comparative effectiveness, in which different treatment paradigms are evaluated for their effect on survival. The most commonly utilized datasets in the US include the Surveillance, Epidemiology, and End Results (SEER) database, SEER-Medicare, and the National Cancer Database (NCDB).

In this article, we will review database publications on pancreatic cancer. We will discuss the differences in how researchers are using their databases and how these publications may affect clinical practice. We will further discuss the inherent difficulties of using large datasets in comparative effectiveness research questions in pancreatic cancer and thus their utility in shaping clinical practice.
Finally, we will discuss future directions for research involving large databases.

\section{Methods and materials}

A combination of Google Scholar, PubMed, and MEDLINE were used with search terms "pancreatic cancer" + "resectable" + "national cancer database", "pancreatic cancer" + "unresectable" + "national cancer database" and more broadly "borderline resectable pancreatic cancer", "locally advanced pancreatic cancer", "unresectable pancreatic cancer", and "resectable pancreatic cancer". Original articles and abstracts from this search were included. Guidelines, including American and European, were reviewed, as well as the randomized clinical trials and single-institution data cited in those guidelines.

The SEER database includes information from 20 registries, representing approximately $28 \%$ of the US population (4). Data available through SEER includes demographics (race and ethnicity, age at diagnosis, month and year of diagnosis, gender, marital status, state and county of residence at time of diagnosis), tumor site and morphology, stage at diagnosis, first course of treatment (surgery, radiotherapy, and more recently systemic therapy), and survival (including cause of death). Local control data and quality of life/treatment complication data are not available in SEER. Specific information on radiotherapy (timing, dose, modality) and systemic therapy (utilization and timing) can now be obtained with an additional data use agreement (4). SEER-Medicare provides linked data from the SEER registry and Medicare administrative claims database and covers the majority of the US population age 65 and older. Thus, information available includes all SEER information listed above in addition to all billable codes to include: tests, procedures, office visits, admissions, durable medical equipment, home health, hospice care, and prescription drugs (5). Medical diagnosis and billing codes can add additional granularity to SEER information. The NCDB is a joint program of the Commission on Cancer $(\mathrm{CoC})$ and the American Cancer Society, which includes data from approximately 1,500 hospitals and clinics in the US and its territories. This database captures nearly $70 \%$ of new cancer diagnoses made in the US (6) but is not population-based. Demographics such as age, race, gender, state, zip code, income and percent of residents without a high school diploma, distance from treatment facility, urban density, treating facility type (academic, 
community, integrated), and primary payer are included. Charlson comorbidity score is included, as well as tumor size, site, grade, cancer stage, and various cancer sitespecific codes that are used for decision-making (i.e., vascular involvement and carbohydrate antigen 19-9 (CA 19-9) level at diagnosis in pancreatic cancer). Information on surgery (number of days from diagnosis, site, surgery type/extent, number of lymph nodes taken/positive, location of lymph nodes, margin status), radiotherapy (number of days from diagnosis, modality, dose, number of treatments), and systemic therapy (number of days from diagnosis, utilization, timing, and number of agents) is available. Survival time is available, as well as 30- and 90-day mortality, if surgery is performed. Unlike SEER, cause of death is not listed in the NCDB. Reasons for refusal of surgery, radiotherapy, and chemotherapy are given for the majority of patients.

\section{Results}

\section{Timing of additional therapy in resectable pancreas cancer}

Given the relatively poor survival of even the most favorable patients who proceed with upfront surgery, the role for NAT in resectable pancreatic cancer is currently an area of inquiry. There has never been a clinical trial comparing NAT to AT, though a meta-analysis did report favorable outcomes from multiple phase II datasets in NAT patients (7).

Multiple NCDB studies have been published investigating the role for NAT in resected disease. Mokdad and colleagues compared clinical stages I-II pancreatic cancer patients treated with NAT to those treated with AT in the NCDB and found a five-month median survival benefit (26 versus 21 months, HR 0.83, $\mathrm{P}<0.01$ ) for patients treated with NAT (8). Additionally, they found pathologic down-staging in terms of T-stage, N-stage, as well as a decreased positive margin rate, though findings were not stratified by stage. Similar findings were reported by Mirkin and colleagues, though only clinical stages II and III patients (not stage I) benefitted from NAT (9). de Geus and colleagues also reported a stage-dependent benefit for NAT (10). Youngwirth and colleagues reported similar findings in terms of pathologic downstaging, but they did not report long-term survival (11). These studies all suggest the possibility of a stage-dependent benefit for NAT in resectable pancreatic cancer.

The role for tumor markers in the treatment of anatomically resectable patients is also increasingly being investigated in pancreatic cancer. In a recent NCDB analysis, Bergquist and colleagues found that increased CA 19-9 levels in resectable patients was an indicator of increased mortality compared to non-secretors or those with normal levels $(\leq 37 \mathrm{U} / \mathrm{mL})(12)$. In these patients, termed "biologically borderline resectable", there was a nonstatistically significant trend for neoadjuvant chemotherapy in mitigating this increased mortality $(\mathrm{P}=0.11)$. Thus, the authors concluded that in anatomically resectable patients, those with an elevated CA 19-9 may be best treated as BRPC (neoadjuvant systemic therapy followed by resection) as opposed to upfront resection. Though only $25 \%$ of patients in the authors' query had CA 19-9 levels reported, this report is concordant with those of others who have found that elevated CA 19-9 levels are associated with poorer outcomes, and thus CA 19-9 is likely a marker of a biologically more aggressive tumor. This NCDB analysis is most interesting, however, because it suggested a possible mitigation for the elevated mortality risk, which has not been reported in the randomized data.

\section{The role for radiotherapy in resectable pancreas cancer}

Three SEER studies have found benefit to post-operative radiotherapy in patients with resected pancreatic cancer, and one is cited in NCCN guidelines as a consideration for utilizing post-operative radiotherapy $(13,14)$. Three NCDB studies, reported between 2013 and 2017, did find modest median survival benefits to the use of postoperative radiotherapy. Kooby and colleagues [2013] (15) found that for resected pancreatic cancer, only adjuvant chemoradiotherapy (HR 0.70, 95\% CI, 0.61-0.80), not chemotherapy alone (HR 1.04, 95\% CI, 0.93-1.18) improved survival over surgical resection. This study is cited in NCCN guidelines as a contrasting study to ESPAC-1, along with a retrospective single-institution series (14). Rutter and colleagues [2015] found that the addition of radiotherapy to adjuvant chemotherapy improved both median and 5 -year overall survival (19.6\% versus $16.5 \%$, $\mathrm{P}<0.001)$ for $\mathrm{p}$ T1-3N0-1 resected pancreatic cancers (16). Finally, Ahmed and colleagues examined stage II patients (T3N0 or T1-3N1), and found a statistically significant survival benefit for median and three-year survival for all AT compared to no AT, with chemoradiotherapy yielding the largest benefit (17). However, at 5 and 10 years, survival was equivalent or worse for chemoradiotherapy compared to no adjuvant therapy. 


\section{The role for and advances in radiotherapy in LAPC}

The role for radiotherapy in LAPC remains controversial. The lack of a "standard of care" treatment regimen is reflected in the NCCN guideline, which allows for firstline treatment with chemotherapy alone, induction chemotherapy followed by chemoradiation, or upfront chemoradiation, either conventionally fractionated or stereotactic body radiotherapy (SBRT) (14). European guidelines give an "I, A" recommendation only to 6 months of gemcitabine and a "minor role of chemoradiation" (18).

Two recently published NCDB analyses compared chemotherapy versus chemoradiotherapy in LAPC, both finding a modestly improved one- and two-year survival with the addition of radiotherapy to chemotherapy $(19,20)$. Additionally, Amini and colleagues found a survival benefit on subgroup analysis with the use of chemotherapy and IMRT, though no significant benefit was observed with chemotherapy and 3D conformal radiotherapy compared to chemotherapy alone (20). Torgeson and colleagues found that multi-agent induction chemotherapy followed by radiotherapy was associated with improved survival compared to multi-agent chemotherapy alone (19). Multiagent chemotherapy alone, however, was superior to singleagent concurrent chemoradiotherapy. These data suggest that the choice of systemic therapy and timing of therapy plays a potentially important role in the efficacy of local therapy, which is concordant with the findings of the most recent meta-analysis. Dose escalation above 54 Gray was not associated with a survival benefit on either univariate or multivariate analysis in the Torgeson data. The Amini data did not directly compare dose but did find that conformality was relevant to survival. That is, patients who underwent the most conformal treatment had a survival benefit compared to chemotherapy alone. These two datasets, when taken together, suggest that dose escalation itself is not likely to be effective unless it can be conformal enough to spare critical organs and prevent toxicity, and local control may only be effective when systemic control is adequately addressed.

SBRT in LAPC has gained traction as another local treatment option either in lieu of or in addition to standard chemotherapy. Zhong and colleagues compared SBRT (4 Gray or more fraction size) to conventionally fractionated radiotherapy (2 Gray or less fraction size) in cT2-T4 N0-N1 pancreatic adenocarcinomas using NCDB (21). They found a survival benefit to SBRT (HR 0.84, $\mathrm{P}<0.001$ ), despite a higher percentage of the
SBRT patients not receiving chemotherapy compared to conventionally fractionated patients $(12.7 \%$ versus $3.9 \%, \mathrm{P}<0.001)$. The survival benefit was increased when all patients had received chemotherapy (HR 0.77, $95 \%$ CI, 0.69-0.85), further suggesting the importance of systemic therapy in providing an opportunity for local treatment to be effective. de Geus and colleagues reported similar findings in NCDB, where SBRT was compared to chemotherapy alone, conventional external beam radiotherapy (3D conformal), and IMRT (22). Prior to matching, SBRT had the highest median survival (13.9 months). After matching, survival with SBRT was superior to chemotherapy alone or $3 \mathrm{D}$ conformal radiation therapy, but equivalent to IMRT. Both of these studies suggest a role for dose escalation, either conventionally with the conformality provided by using IMRT or other such techniques, or through hypofractionation or extreme hypofractionation, which have both been reported in single institution series.

\section{The role for surgical resection in $L A P C$}

Level I evidence does not exist as to the feasibility of resection, either upfront or after a course of non-surgical therapy in LAPC. Given that an R0-R1 surgical resection is considered the most beneficial therapy in pancreatic cancer, more attention has been given to the possibility of "resecting the unresectable" and several single-institutional studies have been published with promising results.

Large hospital datasets can be useful in examining a possible role for surgical resection after primary treatment in LAPC. The Torgeson dataset found that up to $10 \%$ of LAPC patients underwent resection after treatment with multi-agent chemotherapy and chemoradiotherapy, and of those close to $90 \%$ were R0 resections (19). Survival in patients who underwent surgical resection was double that of patients who underwent chemotherapy or chemoradiotherapy alone. Similar findings were reported in the Zhong SBRT dataset, and surgical patients in that dataset experienced $1 / 3$ the risk of death as non-surgical patients (21). Shubert and colleagues (23) examined NCDB for clinical stage III (T4) patients, who by definition are either BRPC or LAPC, and found that resection was possible in $70 \%$ of patients treated with chemotherapy or chemoradiotherapy upfront with the intent to undergo surgical resection, and NAT was associated with a significant survival benefit (MS 20.7 versus 13.7 months, $\mathrm{P}<0.001$ ). 


\section{International use of population database studies}

Datasets from outside the US have been valuable in reporting the incidence and mortality of pancreatic cancer in their country or network of countries. Examples include the Danish series, which found a rising incidence of pancreatic cancer in Denmark over the last 20 years (24). The French national hospital database also found a rising incidence of pancreatic cancer between 2010 and 2014 (25). Rising incidence and unchanged long-term (2-5 years) mortality through 2006 was also described by Klint and colleagues using the NORDCAN database, which was representative of national cancer databases in the Nordic countries (26). The Taiwanese databases (27) also describe a rising incidence of pancreatic cancer. Finally, the SUDCAN and EUROCARE-5 databases were used to describe survival trends from six European countries (Belgium, France, Italy, Spain, Switzerland, Portugal) over the last few years. While there were mild improvements in short-term (18 months and less) survival (28), long-term survival remained dismal $(<5 \%)$.

Comparative effectiveness studies of large datasets outside the US have also added value to the literature. In Taiwan, for example, the use of complementary Chinese herbal medicine in addition to standard therapy was compared to standard therapy alone in patients with pancreatic cancer via the Taiwanese Registry for Catastrophic Illness Patients Database [1997-2010] and found a survival benefit for patients using complementary Chinese herbal medicine (29). Both Korea (Korean Central Cancer Registry and the National Health Insurance Service of the Republic of Korea) (30) and the Netherlands (Netherlands Comprehensive Cancer Organization and PHARMO Database Network) (31) have used their databases to investigate a possible role for Metformin in improving survival in pancreatic patients with concomitant type 2 diabetes. The Swiss examined the role of hospital volume on pancreatic cancer mortality, and found that lower hospital volume was associated with increased mortality after pancreatectomy (32). Finally, the Korean Health Insurance Claims Database has been used to examine the effectiveness of erlotinib when added to gemcitabine for pancreatic cancer, and found that the addition of erlotinib did not improve outcomes compared to gemcitabine alone (33).

\section{Discussion}

\section{Comparing large database results with other published data}

Many published aggregated dataset studies seem to disagree with findings from randomized clinical trials in both resectable and LAPC, where such trials exist. This is true in the role for radiotherapy after resection in resectable disease, and the role for chemoradiotherapy compared to chemotherapy alone in LAPC. There is no level I evidence to compare for the role of neoadjuvant therapy in resectable disease, SBRT in LAPC, or surgery in LAPC. Neoadjuvant therapy seems to be associated with a survival benefit compared to AT in large datasets. One of the caveats of this data is the difficulty of controlling for attrition. That is, the patients who received NAT did not develop metastatic disease or progress prior to surgical resection, and therefore were likely an enriched population by the time they completed surgery.

In terms of post-operative radiotherapy in resectable disease, both SEER and NCDB studies have found a survival benefit to post-operative radiotherapy, though in two separate meta-analyses of randomized trials $(34,35)$, chemoradiotherapy was found to be no better than chemotherapy alone. It is unclear why all three large datasets would conflict with randomized data. It could be argued that the healthiest patients were selected for the addition of radiotherapy in the observational studies. It could also be argued that the chemoradiotherapy group consisted of higher-risk patients who were more likely to benefit from post-operative radiotherapy (positive lymph nodes, margins, bigger tumors), though ideally these factors would be accounted for via either propensity-score matching or multivariate analysis. As the emergence of biomarkers that may indicate more locally aggressive disease, a population who clearly benefits from postoperative radiation therapy may emerge.

In LAPC, the most appropriate primary treatment option remains controversial. Both American and European guidelines cite mixed data on whether survival is affected by the addition of radiotherapy to chemotherapy, with three meta-analyses showing no survival benefit to chemoradiotherapy compared to chemotherapy alone. The first meta-analysis, published in 2007 , cited two trials that compared chemotherapy with chemoradiotherapy and did not find a survival benefit to chemoradiotherapy, though the confidence interval was wide (36). A 2013 meta-analysis found on sub-group analysis that chemoradiotherapy was no better than chemotherapy alone for survival, though it was better than radiotherapy alone (37). A more recent [2018] meta-analysis of 41 prospective studies and close to 2,000 patients also found that in the overall cohort, chemoradiation did not improve 1-year 
survival compared to chemotherapy alone, unless induction chemotherapy was given for at least three months prior to chemoradiotherapy (38). Chemoradiation was also associated with improved survival if maintenance chemotherapy was given, if the chemoradiation was fluorouracil-based, and if the individual study was conducted after 2010 (38). Thus, it does appear that optimizing systemic therapy may play a role in making local control more relevant to survival. The findings of LAP-07, which did not find a survival benefit to chemoradiotherapy compared to chemotherapy alone (39), added to a history of mixed results from studies comparing chemotherapy to chemoradiotherapy in LAPC, though it has been criticized for its use of single-agent gemcitabine, as compared to the multi-agent regimens that have shown improved outcomes in the metastatic setting $(40,41)$.

In terms of resection after primary treatment for LAPC, single-institution studies have increasingly demonstrated a benefit in well selected patients (42-46), which seems to be in agreement with NCDB data. Both large datasets report a lower number of surgical resections (10\%) compared to single-institution reports (20-50\%). It is unlikely that a randomized clinical trial will occur, thus the combination of single institutional data and large database data may be the only guidance available for these patients.

\section{Criticisms of large datasets}

There are many potential criticisms of large aggregated dataset analyses, which have limited their inclusion in treatment guidelines. Such criticisms include: lack of granularity in diagnosis, treatment, and outcomes-related data compared to properly administered prospective trials, as well as cross-over between treatment arms that cannot be accounted for, and concerns over the quality of data represented. Whereas randomized clinical trials employ strict and thorough inclusion and exclusion criteria to rule out interference from other covariates, and crossover between arms is strictly reported, large datasets lack such granularity and therefore are subject to significant confounding.

In any study, confounding is possible through both known and unknown patient and treatment-related factors. Known factors can be adjusted for using various statistical methods, though unknown factors remain limiting. Unknown factors include patient status at diagnosis, such as performance status, specific medical comorbidities, individual socioeconomic status, and treatment intent (definitive versus palliative). Treatment-related factors may lack granularity compared to clinical trials. Finally, outcomes are difficult to interpret, as commonly used datasets lack either cause of death (NCDB) and/or local control data (SEER/NCDB). A commonly cited concern in comparative effectiveness studies is that overall healthier patients tend to undergo more aggressive treatments, and that improved survival seen with more aggressive treatments may be more of a sign of the patients' overall health at diagnosis and tolerance of treatment rather than the treatment itself.

The majority of the datasets do not track a patient's progress through treatment and therefore may not account for cross-over between arms in comparative effectiveness studies. This would mostly be relevant in cases with a treatment sequence question. In LAPC, for example, patients given induction chemotherapy with the intent for chemoradiotherapy may develop either a toxicity or disease progression that disqualified them from receiving radiotherapy. These patients may comprise a significant portion of the chemotherapy-only arm, leaving an enriched patient population in the chemoradiotherapy arm. If the two are compared statistically, there may be a survival benefit that persists through advanced statistical techniquesbut would ultimately be secondary to attrition and failure to account for it.

Concerns over the accuracy of data reporting lead some to question the validity of aggregated datasets. These datasets do undergo rigorous electronic automated checks, which alerts registrars to incomplete or inconsistent data $(6,47)$. However, disconcerting reports have emerged showing significant errors within certain datasets. A recently reported study on thyroid cancer found that up to $40 \%$ of thyroid lobectomies were coded incorrectly in the NCDB (48). Reports like this make it very difficult to hold large dataset results to the same level as clinical trial data, where central review of everything from diagnostic measures (imaging, pathology, cytology, etc.) to the radiotherapy and systemic treatment plans, is often mandatory and reported in the dataset.

In addition to concerns over internal validity in large datasets, external validity may also be questioned. Though the large size of these datasets does lend power and theoretically should be representative of a diverse population, there are often groups that are minimally represented in certain datasets. In the US, for example, NCDB datasets are collected from CoC-accredited facilities, and though $70 \%$ of new cancer diagnoses are captured, there is variable representation across different 
geographic areas and within different sociodemographic groups, thus potentially limiting applicability to the general population (47). Data from SEER-Medicare, while potentially more granular, is representative of only patients 65 and older and thus difficult to extrapolate to younger populations.

\section{Moving forward}

Many of the reported studies in this paper provide an opportunity to define different populations who may benefit from an intervention that is currently not clearly standard of care, though incorporating these studies into prospective trials and then into clinical practice is still challenging. Though there are significant, inherent limitations to using large datasets to define clinical practices, there are options available to improve these databases for the future so that comparative effectiveness and other studies may have more of a role in shaping clinical practice, as opposed to simply generating clinical trial questions. Performance status at diagnosis (standardized across reporting centers), specific medical comorbidities, treatment-related toxicity, disease progression during treatment, local control, and cause of death data would be welcome additions to these datasets. Reasons for not undergoing treatment also could be further defined and more specific. Regular reporting of quality assurance data through random sampling of patients within the dataset matched to their institutional data would relieve the burden of questionable quality.

\section{Conclusions}

The US has recently witnessed a growing number of comparative effectiveness studies completed with large datasets. Within their limitations, these studies have helped elucidate certain best practices in the treatment of pancreatic cancer. They serve an important role in hypothesis-generation, demonstrating outcomes across nonselected populations, and determining effectiveness when prospective data may be unattainable. When taken together, and from a distance, certain themes emerge including the importance of systemic therapy, and the importance of conformality in radiotherapy, that are consistent with both single-institution data and clinical trials. In addition, national and international databases have been valuable in demonstrating trends in survival and care, and increasingly for comparative effectiveness.

\section{Acknowledgements}

The authors would like to thank Michelle Denney for her assistance in editing and formatting.

\section{Footnote}

Conflicts of Interest: S Lloyd has received funding from Sirtex Medical outside of the submitted work. The other authors have no conflicts of interest to declare.

\section{References}

1. Society AC. Key statistics for pancreas cancer. 2016.

2. Siegel RL, Miller KD, Jemal A. Cancer Statistics, 2017. CA Cancer J Clin 2017;67:7-30.

3. Rahib L, Smith BD, Aizenberg R, et al. Projecting cancer incidence and deaths to 2030: the unexpected burden of thyroid, liver, and pancreas cancers in the United States. Cancer Res 2014;74:2913-21.

4. Surveillance, Epidemiology, and End Results Program, Overview of the SEER Program. 2017. Available online: https://seer.cancer.gov/about/overview.html

5. National Cancer Institute, Healthcare Delivery Research Program. 2017.

6. Bilimoria KY, Stewart AK, Winchester DP, et al. The National Cancer Data Base: a powerful initiative to improve cancer care in the United States. Ann Surg Oncol 2008;15:683-90.

7. Gillen S, Schuster T, Meyer Zum Buschenfelde C, et al. Preoperative/neoadjuvant therapy in pancreatic cancer: a systematic review and meta-analysis of response and resection percentages. PLoS Med 2010;7:e1000267.

8. Mokdad AA, Minter RM, Zhu H, et al. Neoadjuvant Therapy Followed by Resection Versus Upfront Resection for Resectable Pancreatic Cancer: A Propensity Score Matched Analysis. J Clin Oncol 2017;35:515-22.

9. Mirkin KA, Hollenbeak CS, Wong J. Survival impact of neoadjuvant therapy in resected pancreatic cancer: A Prospective Cohort Study involving 18,332 patients from the National Cancer Data Base. Int J Surg 2016;34:96-102.

10. de Geus SWL, Eskander MF, Bliss LA, et al. Neoadjuvant therapy versus upfront surgery for resected pancreatic adenocarcinoma: A nationwide propensity score matched analysis. Surgery 2017;161:592-601.

11. Youngwirth LM, Nussbaum DP, Thomas S, et al. Nationwide trends and outcomes associated with neoadjuvant therapy in pancreatic cancer: An analysis of 18 
243 patients. J Surg Oncol 2017;116:127-32.

12. Bergquist JR, Puig CA, Shubert CR, et al. Carbohydrate Antigen 19-9 Elevation in Anatomically Resectable, Early Stage Pancreatic Cancer Is Independently Associated with Decreased Overall Survival and an Indication for Neoadjuvant Therapy: A National Cancer Database Study. J Am Coll Surg 2016;223:52-65.

13. Opfermann KJ, Wahlquist AE, Garrett-Mayer E, et al. Adjuvant radiotherapy and lymph node status for pancreatic cancer: results of a study from the Surveillance, Epidemiology, and End Results (SEER) Registry Data. Am J Clin Oncol 2014;37:112-6.

14. Network NCCN. Pancreatic Adenocarcinoma. 2017.

15. Kooby DA, Gillespie TW, Liu Y, et al. Impact of adjuvant radiotherapy on survival after pancreatic cancer resection: an appraisal of data from the national cancer data base. Ann Surg Oncol 2013;20:3634-42.

16. Rutter CE, Park HS, Corso CD, et al. Addition of radiotherapy to adjuvant chemotherapy is associated with improved overall survival in resected pancreatic adenocarcinoma: An analysis of the National Cancer Data Base. Cancer 2015;121:4141-9.

17. Ahmed A, Walters RW, Malouff TD, et al. Adjuvant therapy in stage II pancreatic cancer: A National Cancer Database analysis. J Clin Oncol 2017;35:283.

18. Ducreux M, Cuhna AS, Caramella C, et al. Cancer of the pancreas: ESMO Clinical Practice Guidelines for diagnosis, treatment and follow-up. Ann Oncol 2015;26 Suppl 5:v56-68.

19. Torgeson A, Lloyd S, Boothe D, et al. Multiagent induction chemotherapy followed by chemoradiation is associated with improved survival in locally advanced pancreatic cancer. Cancer 2017;123:3816-24.

20. Amini A, Jones BL, Stumpf P, et al. Patterns of Care for Locally Advanced Pancreatic Adenocarcinoma Using the National Cancer Database. Pancreas 2017;46:904-12.

21. Zhong J, Patel K, Switchenko J, et al. Outcomes for patients with locally advanced pancreatic adenocarcinoma treated with stereotactic body radiation therapy versus conventionally fractionated radiation. Cancer 2017;123:3486-93.

22. de Geus SWL, Eskander MF, Kasumova GG, et al. Stereotactic body radiotherapy for unresected pancreatic cancer: A nationwide review. Cancer 2017;123:4158-67.

23. Shubert CR, Bergquist JR, Groeschl RT, et al. Overall survival is increased among stage III pancreatic adenocarcinoma patients receiving neoadjuvant chemotherapy compared to surgery first and adjuvant chemotherapy: An intention to treat analysis of the National Cancer Database. Surgery 2016;160:1080-96.

24. Weble TC, Bjerregaard JK, Kissmeyer P, et al. Incidence of pancreatic cancer in Denmark: 70 years of registration, 1943-2012. Acta Oncol 2017;56:1763-8.

25. Maire F, Cibot JO, Compagne C, et al. Epidemiology of pancreatic cancer in France: descriptive study from the French national hospital database. Eur J Gastroenterol Hepatol 2017;29:904-8.

26. Klint A, Engholm G, Storm HH, et al. Trends in survival of patients diagnosed with cancer of the digestive organs in the Nordic countries 1964-2003 followed up to the end of 2006. Acta Oncol 2010;49:578-607.

27. Tseng CM, Huang SP, Liao WC, et al. Incidence and mortality of pancreatic cancer on a rapid rise in Taiwan, 1999-2012. Cancer Epidemiol 2017;49:75-84.

28. Bouvier AM, Bossard N, Colonna M, et al. Trends in net survival from pancreatic cancer in six European Latin countries: results from the SUDCAN population-based study. Eur J Cancer Prev 2017;26 Trends in cancer net survival in six European Latin Countries: the SUDCAN study:S63-9.

29. Kuo YT, Liao HH, Chiang JH, et al. Complementary Chinese Herbal Medicine Therapy Improves Survival of Patients With Pancreatic Cancer in Taiwan: A Nationwide Population-Based Cohort Study. Integr Cancer Ther 2018;17:411-22.

30. Jang WI, Kim MS, Kang SH, et al. Association between metformin use and mortality in patients with type 2 diabetes mellitus and localized resectable pancreatic cancer: a nationwide population-based study in korea. Oncotarget 2017;8:9587-96.

31. Frouws MA, Sibinga Mulder BG, Bastiaannet E, et al. No association between metformin use and survival in patients with pancreatic cancer: An observational cohort study. Medicine (Baltimore) 2017;96:e6229.

32. Guller U, Warschkow R, Ackermann CJ, et al. Lower hospital volume is associated with higher mortality after oesophageal, gastric, pancreatic and rectal cancer resection. Swiss Med Wkly 2017;147:w14473.

33. Shin S, Park CM, Kwon H, et al. Erlotinib plus gemcitabine versus gemcitabine for pancreatic cancer: realworld analysis of Korean national database. BMC Cancer 2016;16:443.

34. Butturini G, Stocken DD, Wente MN, et al. Influence of resection margins and treatment on survival in patients with pancreatic cancer: meta-analysis of randomized controlled trials. Arch Surg 2008;143:75-83; discussion 83. 
35. Stocken DD, Buchler MW, Dervenis C, et al. Metaanalysis of randomised adjuvant therapy trials for pancreatic cancer. Br J Cancer 2005;92:1372-81.

36. Sultana A, Tudur Smith C, Cunningham D, et al. Systematic review, including meta-analyses, on the management of locally advanced pancreatic cancer using radiation/combined modality therapy. Br J Cancer 2007;96:1183-90.

37. Chen Y, Sun XJ, Jiang TH, et al. Combined radiochemotherapy in patients with locally advanced pancreatic cancer: a meta-analysis. World J Gastroenterol 2013;19:7461-71.

38. Chang JS, Chiu YF, Yu JC, et al. The Role of Consolidation Chemoradiotherapy in Locally Advanced Pancreatic Cancer Receiving Chemotherapy: An Updated Systematic Review and Meta-Analysis. Cancer Res Treat 2018;50:562-74.

39. Hammel P, Huguet F, van Laethem JL, et al. Effect of Chemoradiotherapy vs Chemotherapy on Survival in Patients With Locally Advanced Pancreatic Cancer Controlled After 4 Months of Gemcitabine With or Without Erlotinib: The LAP07 Randomized Clinical Trial. JAMA 2016;315:1844-53.

40. Conroy T, Desseigne F, Ychou M, et al. FOLFIRINOX versus gemcitabine for metastatic pancreatic cancer. $\mathrm{N}$ Engl J Med 2011;364:1817-25.

41. Von Hoff DD, Ervin T, Arena FP, et al. Increased survival in pancreatic cancer with nab-paclitaxel plus gemcitabine. N Engl J Med 2013;369:1691-703.

42. Cloyd JM, Katz MH, Prakash L, et al. Preoperative

Cite this article as: Torgeson A, Tao R, Garrido-Laguna I, Willen B, Dursteler A, Lloyd S. Large database utilization in health outcomes research in pancreatic cancer: an update. J Gastrointest Oncol 2018;9(6):996-1004. doi: 10.21037/ jgo.2018.05.15
Therapy and Pancreatoduodenectomy for Pancreatic Ductal Adenocarcinoma: a 25-Year Single-Institution Experience. J Gastrointest Surg 2017;21:164-74.

43. Ferrone CR, Marchegiani G, Hong TS, et al. Radiological and surgical implications of neoadjuvant treatment with FOLFIRINOX for locally advanced and borderline resectable pancreatic cancer. Ann Surg 2015;261:12-7.

44. Hitchcock KE, Nichols RC, Morris CG, et al. Feasibility of pancreatectomy following high-dose proton therapy for unresectable pancreatic cancer. World J Gastrointest Surg 2017;9:103-8.

45. Murata Y, Usui M, Hayasaki A, et al. Clinical significance of conversion surgery after gemcitabine plus S-1 based chemoradiotherapy in patients with initially locally advanced unresectable pancreatic adenocarcinoma: application of intratumoral expression of Tenascin $\mathrm{C}$ as a surrogate marker. Pancreatology 2016;16:S33.

46. Dean A, Higgs D, Yusoff I, et al. P-208Resecting the unresectable - long course gemcitabine/nab-paclitaxel followed by chemoradiation to downstage locally advanced pancreatic adenocarcinoma. Ann Oncol 2017;28:mdx261.06-mdx.06.

47. Boffa DJ, Rosen JE, Mallin K, et al. Using the National Cancer Database for Outcomes Research: A Review. JAMA Oncol 2017;3:1722-8.

48. Thoburn KK, Gress DM, Mallin K, et al. Questioning the Quality of Thyroid Cancer Data: Thoughts from the Commission on Cancer and the National Cancer Database. Ann Surg Oncol 2017;24:1157-8. 